\title{
What has been missed for predicting human attention in viewing driving clips?
}

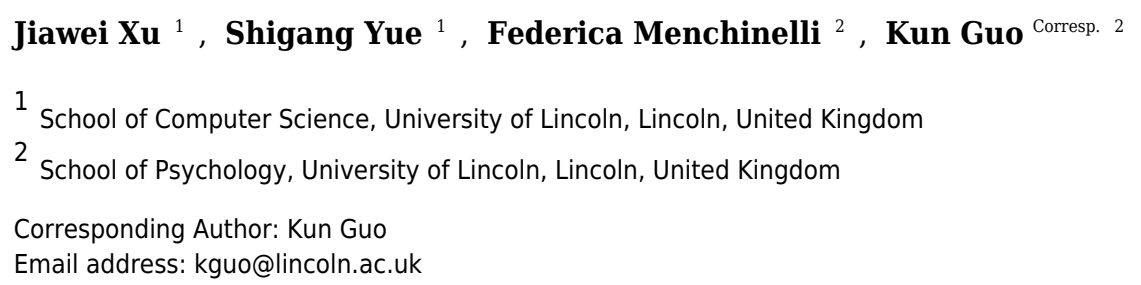

Recent research progress on the topic of human visual attention allocation in scene perception and its simulation is based mainly on studies with static images. However, natural vision requires us to extract visual information that constantly changes due to egocentric movements or dynamics of the world. It is unclear to what extent spatiotemporal regularity, an inherent regularity in dynamic vision, affects human gaze distribution and saliency computation in visual attention models. In this free-viewing eyetracking study we manipulated the spatio-temporal regularity of traffic videos by presenting them in normal video sequence, reversed video sequence, normal frame sequence, and randomised frame sequence. The recorded human gaze allocation was then used as the 'ground truth' to examine the predictive ability of a number of state-of-the-art visual attention models. The analysis revealed high inter-observer agreement across individual human observers, but all the tested attention models performed significantly worse than humans. The inferior predictability of the models was evident from indistinguishable gaze prediction irrespective of stimuli presentation sequence, and weak central fixation bias. Our findings suggest that a realistic visual attention model for the processing of dynamic scenes should incorporate human visual sensitivity to spatiotemporal regularity and central fixation bias. 
$7 \quad$ * Corresponding Author:

8 Email:kguo@lincoln.ac.uk 


\section{Abstract}

11 Recent research progress on the topic of human visual attention allocation in scene

12 perception and its simulation is based mainly on studies with static images. However, natural

13 vision requires us to extract visual information that constantly changes due to egocentric

14 movements or dynamics of the world. It is unclear to what extent spatio-temporal regularity, an

15 inherent regularity in dynamic vision, affects human gaze distribution and saliency computation

16 in visual attention models. In this free-viewing eye-tracking study we manipulated the spatio-

17 temporal regularity of traffic videos by presenting them in normal video sequence, reversed

18 video sequence, normal frame sequence, and randomised frame sequence. The recorded human

19 gaze allocation was then used as the 'ground truth' to examine the predictive ability of a number

20 of state-of-the-art visual attention models. The analysis revealed high inter-observer agreement

21 across individual human observers, but all the tested attention models performed significantly

22 worse than humans. The inferior predictability of the models was evident from indistinguishable

23 gaze prediction irrespective of stimuli presentation sequence, and weak central fixation bias. Our

24 findings suggest that a realistic visual attention model for the processing of dynamic scenes

25 should incorporate human visual sensitivity to spatio-temporal regularity and central fixation 26 bias.

\section{Introduction}

The amount of available visual information in our surroundings is often beyond our 30 brain's processing capability. To effectively interact with our natural and social world, we

31 selectively gaze at and process a limited number of local scene regions or visual items that are

32 informative or interesting to us. As our gaze allocation is a sensitive index of attention, 
33 motivation and preference (Henderson, 2007), the fixated regions tend to have a distinct

34 subjective perceptual quality which enables them to stand out from the neighbours, and the

35 choice of these salient targets reflects our internal representation of the external world.

36 The central research question in this active visual exploration process is to understand

37 how we choose the fixated regions in the scene. Psychological studies have suggested that both

38 bottom-up local saliency computation and top-down cognitive processes are actively involved in

39 determining our fixations in scene exploration (Henderson, 2007). The bottom-up signals include

40 image immanent features that could transiently attract fixation irrespective of a particular task

41 demand. For instance, we tend to avoid low-contrast and homogeneous 'predictable' regions in

42 natural scenes, and bias our fixation to local features with high-contrast, high spatial frequency,

43 high edge density, and complex local structure (e.g., curved lines, edges and corners, as well as

44 occlusions or isolated spots) (Mannan, Ruddock, \& Wooding 1996; Reinagel \& Zador, 1999;

45 Parkhurst \& Niebur, 2003; Acik, Onat, Schumann, Einhauser, \& Konig, 2009), or to local

46 regions deviated from surrounding image statistics (Einhäuser et al., 2006). The top-down

47 signals, such as an individual's expectation, experience, memory, semantic and task-related

48 knowledge, are task-dependent and could significantly modulate our gaze allocation in scene

49 exploration (e.g., Guo, Smith, Powell, \& Nicholls, 2012; Pollux, Hall, \& Guo, 2014; Gavin,

50 Houghton, \& Guo, 2015). Recent studies have further proposed that fixation selection is uniquely

51 driven by learned associations between stimuli and rewards (Anderson, 2013), and is influenced

52 by aspects of innate human bias, such as the tendency to fixate human/animal face and body in

53 the scene and to look more often at the central part of the scene (Tatler, Hayhoe, Land, \& Ballard, 54 2011). 
Motivated by these empirical studies, computer vision scientists have tried to develop

human-vision-inspired foveated active artificial vision systems capable of working in real-time, and have proposed various computational models for predicting and/or mimicking human gaze

58 allocation in natural vision (Borji, Sihite, \& Itti, 2013). Closely resembling our knowledge about neural processing in the early visual system, the widely cited bottom-up saliency model (Itti \& Koch, 2000) compares local image intensity, colour and orientation through centre-surround filtering at eight spatial scales, combines them into a single salience (conspicuity) map with a winner-take-all network and inhibition-of-return, and then produces a sequence of predicted fixations that scan the scene in order of decreasing salience. To improve its relatively low level of predictive power (e.g., $57 \%-68 \%$ correct fixation prediction in some scene free-viewing tasks; Betz, Kietzmann, Wilming, \& Konig, 2010), aspects of innate human bias and top-down processing, such as face or object detection, scene context, natural statistics and learned

67 associations between stimuli and rewards, are later incorporated into the model (e.g., Torralba, 68 Oliva, Castelhano, \& Henderson, 2006; Judd, Ehinger, Durand, \& Torralba, 2009; Kanan, Tong, Zhang, \& Cottrell, 2009; Goferman, Zelnik-Manor, \& Tal, 2012). This combination of bottom-

70 up saliency-driven information and top-down scene understanding has greatly improved gaze prediction in real-word visual search and free-viewing tasks.

However, the experimental findings discussed above and computational visual attention models in scene perception are derived mainly from studies using images in laboratory settings. In the real world we need to select, extract and process visual information that constantly changes due to egocentric movements or dynamics of the world. The motion cues are likely to attract visual attention and play a crucial role in subsequent scene understanding. Indeed, recent psychological studies have shown that motion and continuous temporal change are stronger 
78 predictors for gaze allocation than local image intensity or colour (Carmi \& Itti, 2006; Le Meur,

79 Le Callet, \& Barba, 2007; Dorr, Martinetz, Gegenfurtner, \& Barth, 2010), and can enhance

80 perceptual performance in understanding ambiguous visual cues, such as degraded or subtle

81 facial expressions (Cunningham \& Wallraven, 2009).

82 Currently, most of the visual attention models focus on spatial information processing

83 with little consideration of temporal cues (Borji, Sihite, \& Itti, 2014), especially for the evolved

84 spatial-temporal coherence over a period of time. For example, in the saliency-based visual

85 attention system (Itti, Koch, \& Niebur, 1998), three feature channels - colour, intensity, and

86 orientation, and multi-scale image features are combined into a single topographical saliency

87 map, in order to break down the complex scene understanding problem by rapidly selecting

88 conspicuous locations for the detailed analysis. Attention based on information maximization

89 (AIM) model uses Shannon's self-information measure to calculate the saliency of image regions

90 (Bruce \& Tsotsos, 2006), while the incremental coding length (ICL) approach measures the

91 respective entropy gain of each feature to maximize the entropy of the sampled visual features in

92 both dynamic and static scenes (Hou \& Zhang, 2008). Recently a spatiotemporal saliency

93 algorithm based on a centre-surround framework has been proposed in which the salience map

94 was calculated using dynamic textures (Mahadevan \& Vasconcelos, 2010). Marat's model

95 (Marat et al., 2009) extracts two signals from the video stream corresponding to the two main

96 outputs of the retina: parvocellular and magnocellular pathways, both signals are split into

97 elementary feature maps by cortical-like filters. These maps are then fused into a spatio-temporal 98 saliency map.

99 To further enhance the model's predictive power, we proposed a dynamic visual attention 100 model (Xu, Yue, \& Tang, 2013) based on three channels. The first channel is motion intensity to 
101 reveal the faster moving objects. Then the spatial cues indicate the different moving objects in

102 the spatial location, while the temporal cues denote the variability of one object in the temporal

103 dimension. The rarity factor is imported to describe the unexpected or novel moving object that

104 appeared in the visual field, which is likely to attract visual attention and should be captured or

105 reflected in the saliency map, but to ignore the receding objects. The model has been developed

106 further to analyze the attention shifts by fusing the top-down bias and bottom-up cues (Xu \& Yue,

107 2014). The proposed model has five modules: the pre-learning process, top-down bias, bottom-

108 up mechanism, multi-layer neural network and attention. The motion attention is estimated

109 based on a motion vector field (MVF) comprising of three inductors: intensity inductor, spatial

110 coherence inductor, and temporal coherence inductor (Ma, Hua, Lu, \& Zhang, 2005). Ben et al.

111 (2008) also proposed a biologically motivated dynamic bottom-up selective attention model,

112 which employs the maximum entropy algorithm to analyze the dynamics of the successive static

113 saliency maps.

114 Although some of these models work reasonably well in simulated laboratory conditions,

115 it is unclear how well they could predict human attention allocation in naturalistic conditions.

116 For instance, these models tend to assign a high saliency rating to a moving object regardless of

117 the predictability of motion trajectory, but we are unlikely to attend to a predictable movement

118 (e.g., a travelling car with constant speed and predictable direction) for a prolonged period in

119 natural environments. Given that our visual system is evolved to process dynamic spatiotemporal

120 information, it is reasonable to assume that natural scene dynamics could elicit an optimal gaze

121 strategy to facilitate scene understanding. Among various scene-dependent spatial and temporal

122 cues in natural vision, spatio-temporal regularity or predictability, in which objects around us

123 often occur and move in statistically predictable ways to create a stream of visual inputs which 
124 are spatially and temporally coherent (e.g., the trajectory of a car moving on the motorway or an

125 apple falling from a tree), is an inherent common regularity in the natural environment. Recent

126 psychological and brain imaging studies have shown that the human visual system exploits this

127 spatio-temporal regularity to effectively process dynamic visual inputs, such as detecting and 128 discriminating the moving target (e.g., Guo et al., 2004; Pollux \& Guo, 2009; Hall, Pollux, \&

129 Guo, 2010; Pollux, Hall, Roebuck, \& Guo, 2011; Roebuck, Bourke, \& Guo, 2014).

130 To the best of our knowledge, we are not aware of any visual attention model that has

131 examined the effect of movement predictability on its predictive power or has incorporated this

132 spatio-temporal regularity into saliency computation. In this study, we installed a camera in the

133 car and recorded typical driving scenes with different levels of traffic complexity. To

134 systematically manipulate the videos' spatio-temporal regularity, each clip was presented in four

135 different formats, such as normal video sequence, reversed video sequence, normal image

136 sequence, and randomised image sequence. Such manipulation of the presentation sequence of

137 the videos could selectively disrupt the temporal frame order (i.e. reversed video sequence), the

138 spatial frame coherence (i.e. normal image sequence) or the spatio-temporal frame coherence (i.e.

139 randomised image sequence) of the visual stream inputs. The gaze allocation of human observers

140 viewing these clips was recorded and then used as the 'ground truth' to examine the predictive

141 ability of the state-of-the-art visual attention models.

\section{Methods}

\section{Ethics Statement}

145 The Ethical Committee in School of Psychology, University of Lincoln, approved this

146 study. Written informed consent was obtained from each participant prior to the study, and all 
147 procedures complied with the British Psychological Society Code of Ethics and Conduct. All the

148 relevant measures, conditions and data exclusions have been reported, and the sample size was

149 determined based on our previous research (Röhrbein et al., 2015).

150 Eye-tracking study

151 Thirty-five undergraduate students (27 female, 8 male), age ranging from 18 to 28 years

152 old with a mean of $19.4 \pm 0.37$ (Mean \pm SEM), volunteered to participate in this study. All 153 participants had normal or corrected-to-normal visual acuity, and normal colour vision (checked 154 with Ishihara's Tests for Colour Deficiency, 24 Plates Edition).

155 Visual stimuli were presented on a non-interlaced gamma-corrected colour monitor (30

$156 \mathrm{~cd} / \mathrm{m}^{2}$ background luminance, $100 \mathrm{~Hz}$ frame rate, Mitsubishi Diamond Pro 2070SB) with a 157 resolution of $1024 \times 768$ pixels. At a viewing distance of $57 \mathrm{~cm}$, the monitor subtended a visual 158 angle of $40 \times 30^{\circ}$.

159 Thirty high-definition colour video clips were sampled from the authors' collection of 160 traffic videos, taken by attaching a digital camcorder to the car windscreen. These video clips 161 represent a variety of daily traffic conditions, such as approaching traffic lights, road junction 162 and roundabout; low-speed driving at car park and residential area or on inner-city road; high163 speed driving on single carriageway, dual carriageway and motorway (see Fig.1 for example

164 frames from typical video scenes). Each video clip lasted 3 seconds (30 frames per second) and 165 all clips were identical in size $(720 \times 404$ pixels $)$.

166

$$
\text { A }
$$

B 
167

168

169

170

171

172

173

174

175

176

177

178

179

180
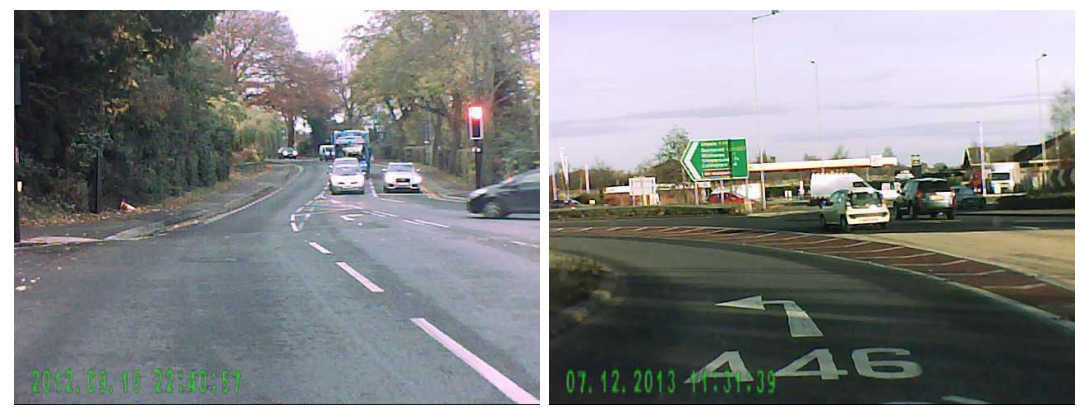

C

D

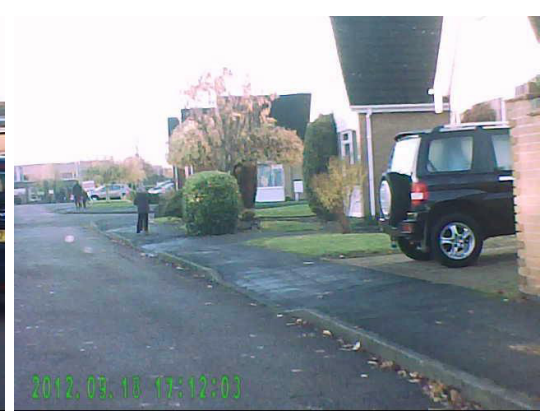

E

F
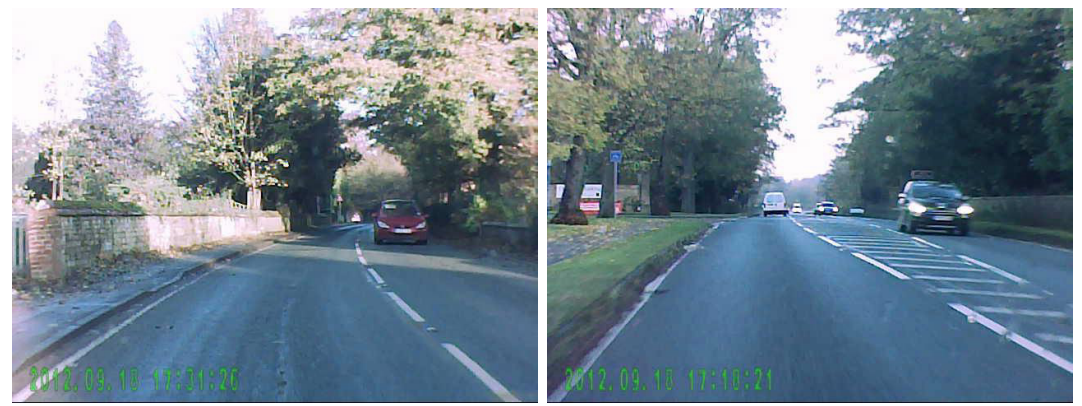

H
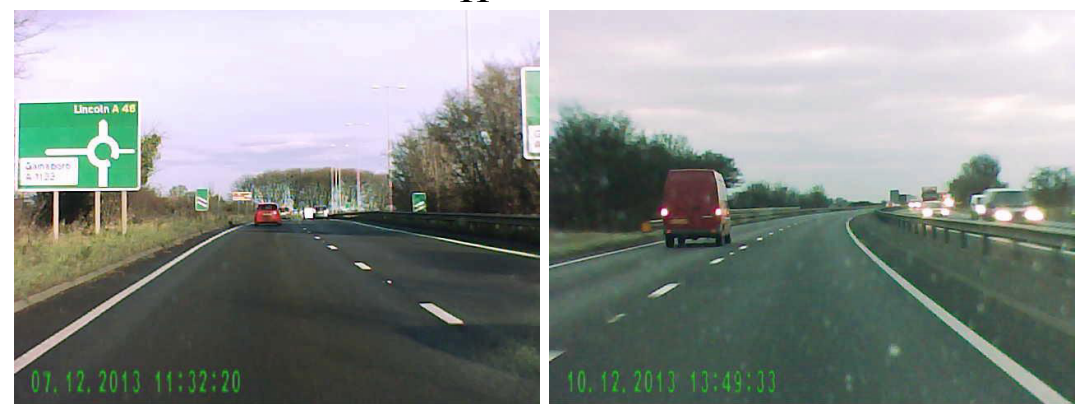

Figure 1. Example frames from traffic videos used in this study. The videos included clips representing approaching traffic lights (A) and a road junction (B), low-speed driving at a car park (C) and residential area (D), driving in an inner-city road (E and F), and high-speed driving in a dual carriageway $(\mathrm{G})$ and motorway $(\mathrm{H})$.

To examine to what extent the spatio-temporal regularity of the videos would affect our gaze allocation, each video clip was manipulated and later presented in four conditions: (1) 
181 normal video sequence: the clip was presented in its original, predictable sequence (from 1st

182 frame to 90th frame); (2) reversed video sequence: the same clip was presented in reverse motion

183 or time-reversed sequence (from 90th frame to 1st frame); (3) normal image sequence: six still

184 shots were sampled at the beginning, middle and end of the video sequence (i.e. 1st, 16th, 31 st,

185 46th, 61st and 76th frame), and then presented in their original order, each still image was

186 displayed for $500 \mathrm{~ms}$; (4) randomised image sequence: the same six still images were presented

187 in a randomised sequence, each lasting $500 \mathrm{~ms}$. As a result, 120 video clips were generated for

188 the testing session (30 original clips $\times 4$ manipulations). These clips were displayed once in a

189 random order during the testing.

190 A free-viewing task was used to mimic natural viewing condition. During the

191 experiments the participants sat in a chair with their head restrained by a chin-rest, and viewed

192 the display binocularly. To calibrate eye movement signals, a small red fixation point (FP, $0.3^{\circ}$

193 diameter, $15 \mathrm{~cd} / \mathrm{m}^{2}$ luminance) was displayed randomly at one of 9 positions $(3 \times 3$ matrix $)$

194 across the monitor. The distance between adjacent FP positions was $10^{\circ}$. The participant was

195 instructed to follow the FP and maintain fixation for 1 second. After the calibration procedure,

196 the trial was started with four FPs presented at the corners of the screen area in which the videos

197 were displayed. To minimize initial central fixation bias in scene viewing (Tatler, 2007), the

198 participant was instructed to randomly choose one FP to fixate. If the participant maintained

199 fixation for 1 second, the FP disappeared and a testing video clip was presented at the centre of

200 the screen. During the free-viewing presentation, the participant was instructed to "view the

201 video clip as you normally do". No reinforcement was given during this procedure. All

202 participants have viewed all of the 120 video clips. 
204 through the Hole-in-Card test or the Dolman method if necessary) were measured using a Video

205 Eyetracker Toolbox (a camera-based system tracking pupil centre and corneal reflection) with 50

$206 \mathrm{~Hz}$ sampling frequency and up to $0.25^{\circ}$ accuracy (Cambridge Research Systems, UK.

$207 \mathrm{http} / /$ crsltd.com/tools-for-vision-science/eye-tracking/high-speed-video-eye-tracker-toolbox/).

208 The software developed in Matlab computed horizontal and vertical eye displacement signals as

209 a function of time to determine eye velocity and position (Guo, Mahmoodi, Robertson, \& Young,

210 2006). The location of gaze point within each video frame was extracted from the raw data.

211

\section{Data analysis and model comparison}

Human gaze allocation in viewing of the normal video sequences was treated as the

214 'ground truth' or 'bench mark' to compare the participants' gaze behaviour on the four

215 presentation conditions and the predictive power of the chosen computational models. The bench

216 mark gaze location at each frame was averaged from 35 participants after checking inter-

217 observer agreement (or individual differences in gaze allocation) through the "all-except-one

218 observers" protocol (i.e. how well the fixation distribution of each participant can be predicted

219 by that of other participants; Röhrbein et al., 2015). We then adopted a frame-based similarity

220 measurement to quantify the differences in comparison. For each frame within a given video

221 sequence, we first calculated the Euclidean Distance $\left(D_{f}\right)$ between bench mark gaze location

$222\left(x_{0}, y_{0}\right)$ and predicted gaze location $\left(x_{1}, y_{1}\right)$ from a chosen computational model.

$$
D_{f}=\sqrt{\left(x_{1}-x_{0}\right)^{2}+\left(y_{1}-y_{0}\right)^{2}}
$$


Then for the same video frame, we used the same equation to calculate the Euclidean

225

226

228

229

230

231

232

233

234

235

236

237

238

239

240

241

242

244 245 246

Distance between bench mark gaze location $\left(x_{0}, y_{0}\right)$ and actual gaze location $\left(x_{1}, y_{1}\right)$ from each participant $\left(\check{D}_{i}\right)$, and computed the averaged Euclidean Distance across all the participants $(\check{D})$.

$$
\check{D}=\frac{1}{35}\left(\sum_{i=1,2, \ldots, 35} \check{D}_{i}\right)
$$

The similarity score $(S)$ for this video frame was then computed as

$$
S=\frac{\check{D}}{\check{D}+D_{f}}
$$

After repeating the same protocol for all the frames within this given video sequence, the final similarity score for the clip was then averaged across all the similarity measures for each frame in the video, and was ranged from 0 to 1 , in which 0 represents no similarity at all and 1 means identical spatial-temporal gaze distribution between human observers and model prediction. This normalized approach for similarity measurement could minimize the impact of stimulus size or content on similarity calculation, and facilitate data comparison across different stimulus conditions, different participant groups or different studies.

Normalized Scanpath Saliency (NSS) and Area under Curve (AUC) or Receiver Operating Characteristics (ROC) are other commonly used measures in the literature to compare the predictive power of visual attention models (Röhrbein, Goddard, Schneider, James, \& Guo, 2015). However, NSS is the average of the response values of human gaze allocation without considering spatial phase (Rothenstein \& Tsotsos, 2006), and the computation of AUC or ROC is more suitable for static image rather than dynamic video analysis (e.g., unable to capture frameby-frame dynamic changes in a model's predictive performance; Green \& Swets, 1966; Zou, O'Malley, \& Mauri, 2007). In this study we therefore focused on comparing similarity score calculated from different presentation conditions with different computational models. As 
247 discussed in the Introduction, very few visual attention models have incorporated motion cues in

248 the saliency computation. Based on their significance in the literature, we chose to compare the

249 predicitive power of six models from Itti et al. (1998), Bruce and Tsotsos (2006), Hou and

250 Zhang (2008), Mahadevan and Vasconcelos (2010), Marat et al. (2009), and Xu et al. (2013).

251 These models have all been constructed for dynamic displays and contain a 'motion' channel.

252 For each tested video clip, its saliency map (or predicted fixation allocation) was computed by

253 these models separately using Matlab programmes with default settings obtained from the model

254 developers.

255

256 Results

257 We first calculated the baseline of human performance using the similarity score, which 258 measures how well the fixation distribution of each participant can be predicted by that of other 259 participants (all-except-one observers). Specifically, we selected one participant's fixation 260 allocations as actual fixations that we wanted to predict, and compared them with the averaged 261 fixation allocations from the remaining participants. By repeating this procedure for all 262 participants and averaging the resulting similarity scores, we obtained a measure for the 263 variability (or inter-observer agreement) within all human gaze patterns that can serve as an 264 upper bound to the performance of a given computational model. As shown in Fig. 2, 265 irrespective of the stimuli presentation sequence, the gaze patterns from individual human 266 participants were highly similar, with a similarity score distributed around 0.83 , suggesting small

267 human individual differences and a reliable 'ground truth' baseline or benchmark for model 268 comparisons. The chance or random baseline was calculated by measuring the similarity score 269 between actual human gaze allocation and randomly selected 'gaze' allocation at each frame for 
270 each video clip and presentation sequence. After repeating the same procedure 100 times, the 271 averaged similarity score varied between 0.46 and 0.49 across different presentation sequences

272 which was significantly below human baseline.

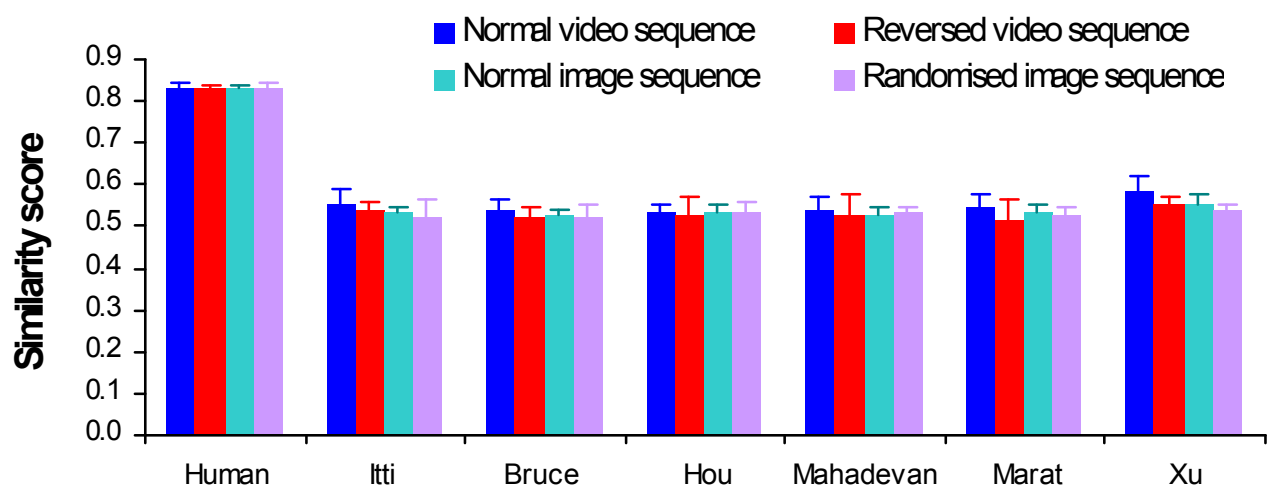

273

274 Figure 2. Similarity scores from human observers and different visual attention models in 275 computing saliency map of traffic videos and images with different presentation sequences. Error 276 bars represent standard deviation.

When compared with the averaged human gaze pattern and chance baseline, the 279 predictive power of the tested models was clearly below the human baseline but was above the 280 chance baseline regardless of presentation sequence (Fig. 2). To systematically compare the 281 models' performance under different stimuli presentation sequences, a 7 (models: human 282 baseline plus 6 visual attention models) $\times 4$ (presentation sequences) repeated-measures analysis 283 of variance (ANOVA) was conducted with similarity score as the dependent variable.

284 Greenhouse-Geisser correction was applied where sphericity was violated. The analysis revealed 285 a significant main effect of model $\left(F(6,174)=1614.88, p<0.001, \eta_{p}{ }^{2}=0.98\right)$ and presentation 286 sequence $\left(F(2.03,58.77)=10.67, p<0.001, \eta_{p}{ }^{2}=0.27\right)$, and a significant interaction between model 287 and presentation sequence $\left(F(6.23,180.67)=4.2, \quad p<0.001, \quad \eta_{p}{ }^{2}=0.13\right)$. Detailed post-hoc 288 comparisons with Bonferroni correction further showed that all the visual attention models 
289 performed significantly worse than human baseline (all $p s<0.01$ ). Among the tested models, the $290 \mathrm{Xu}$ model (Xu et al., 2013) performed slightly better than other models (all $p s<0.01$ ), and the

291 performance from the rest of models was indistinguishable (all $p s>0.05$ ). Regarding stimulus

292 presentation sequence, the normal video sequence induced better model performance for the

293 Bruce (Bruce \& Tsotsos, 2006), Marat (Marat et al., 2009), and Xu (Xu et al., 2013) models (all

$294 p s<0.01)$. All the models demonstrated the same predictive performance for the presentation of 295 reversed video sequence, normal image sequence and randomised image sequence (all $p s>0.01$, 296 Fig. 2).

298

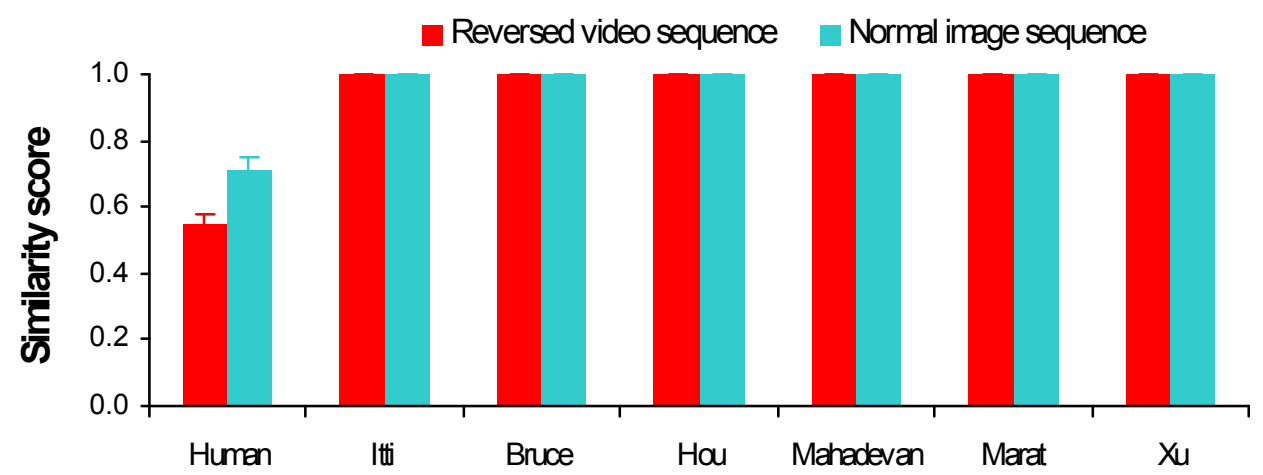

299 Figure 3. Changes in similarity score over different stimulus presentation sequence. For each

300 human observer and each visual attention model, we computed the similarity score between

301 fixation allocations (actual fixation allocations from human observers, and predicted fixation

302 allocations from the models) in the normal video sequence and in the reversed video sequence or 303 in the normal image sequence. Error bars represent standard deviation.

As clearly shown in Fig. 2, the tested computational visual attention models performed

307 poorly across different video presentation sequences when using actual human gaze allocation as

308 the baseline (or bench mark). To understand the potential causes for the models' inferior 
309 performance, we directly examined the impact of presentation sequence manipulation on actual

310 and predicted gaze allocation by using gaze allocation in normal video sequence as the baseline.

311 Specifically, for each human observer and each visual attention model, we computed the

312 similarity score between fixation allocation (actual fixation allocations for human observers, and

313 predicted fixation allocations for the models) in the normal video sequence and in the reversed

314 video sequence, and between fixation allocation in the normal video sequence and in the normal

315 image sequence (Fig. 3). Such analysis could directly inform the extent to which the disruption

316 of temporal frame order (i.e. reversed video sequence) or spatial frame coherence (i.e. normal

317 image sequence) of the visual stream inputs would affect actual and predicted gaze allocation.

318 A 7 (models) $\times 2$ (presentation sequences) ANOVA revealed a significant main effect of

319 model $\left(F(6,174)=7139.36, p<0.001, \eta_{p}{ }^{2}=0.99\right)$ and presentation sequence $(F(1,29)=273.28$,

$320 p<0.001, \eta_{p}{ }^{2}=0.9$ ), and a significant interaction between model and presentation sequence

$321\left(F(6,174)=273.29, p<0.001, \eta_{p}{ }^{2}=0.9\right)$. Specifically, manipulating presentation sequence has

322 clearly decreased similarity between actual human fixation distributions in the normal video

323 sequence and in the reversed video sequence or in the normal image sequence, and such effect

324 was more evident in the reversed video than in the normal image sequence (all $p s<0.01$, Fig. 3),

325 suggesting that temporal frame order might play a more important role in determining human

326 gaze allocation in video-watching than spatial frame coherence. All the visual attention models,

327 on the other hand, have predicted the same location of salient region for each video frame

328 regardless of presentation sequence (i.e. indistinguishable high similarity scores when comparing

329 between normal video sequence and reversed video sequence, or between normal video sequence

330 and normal image sequence), suggesting that the crucial role of temporal frame order has been

331 ignored in these computational models. 
A

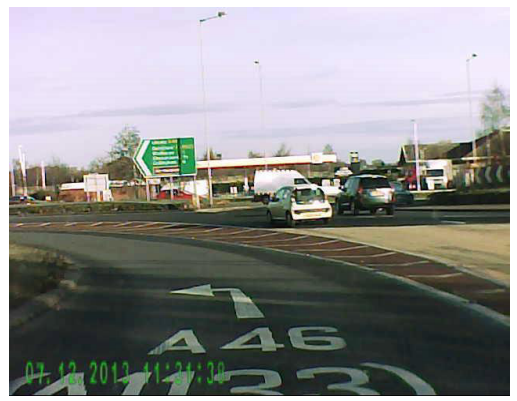

341

342

343

C
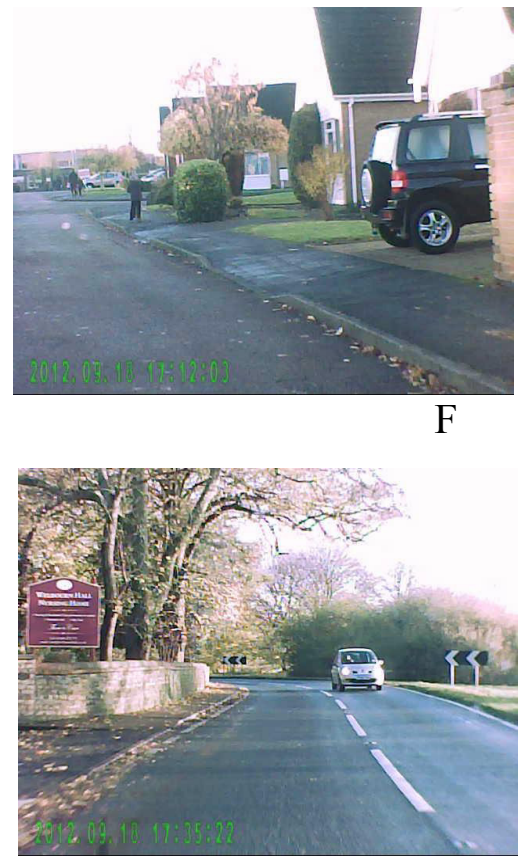

D

B
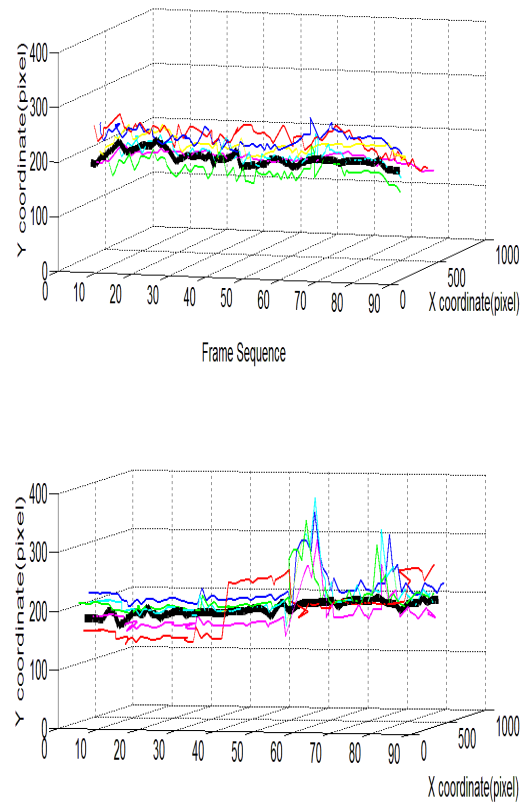

Frame Sequence

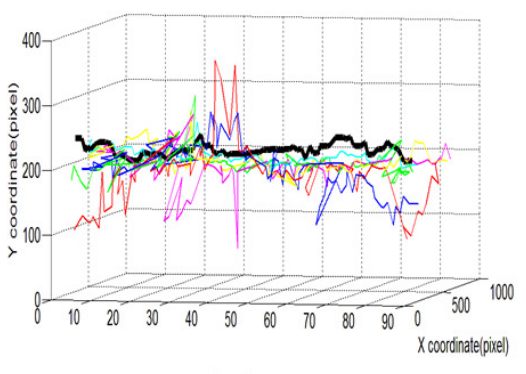

Frame Sequence

346 Figure 4. Distribution of actual and predicted gaze allocation for each frame of three typical 347 videos ( $\mathrm{A}$ and $\mathrm{B}, \mathrm{C}$ and $\mathrm{D}, \mathrm{E}$ and $\mathrm{F}$ ) presented in normal video sequence. The pictures are 
348 example frames extracted from videos representing junction approach (A), low-speed (C) and

349 high-speed driving (E). The black lines in B, D, and F represent the averaged co-ordinates of

350 human gaze allocation for each of the 90 frames. The coloured curves represent predicted gaze

351 allocation from the six tested visual attention models.

352

353

When exploring static images, humans tend to look more frequently at the central part of

354 the image, especially at the initial stage of image viewing (Tatler, 2007; Röhrbein et al., 2015).

355 This central fixation bias is mainly due to the image centre providing a convenient starting point

356 to ensure rapid access to every point of interest in the image (Tatler, 2007). As demonstrated in

357 Fig. 4, human observers seemed to show similar central location bias in video watching.

358 Irrespective of video content, the spatial location of their gaze point was less varied across each

359 frame and was more often close to the frame image centre in comparison with the predicted gaze

360 location from the tested visual attention models. To quantify the difference between the spread of

361 the fixations made from humans and models, for each video we averaged both actual and

362 predicted fixation distance from each frame's image centre (Fig. 5). The one-way ANOVA

363 revealed a significant difference between the actual and predicted fixation distributions

$364\left(F(6,174)=3.35, p=0.004, \eta_{p}{ }^{2}=0.11\right)$. Specifically, humans demonstrated a stronger central

365 fixation bias than the visual attention models (all $p s<0.05$ ), but there was no clear difference in

366 the predicted fixation distance from the image centre across the six tested models (all $p s>0.05$ ).

367 Clearly, this property of human central fixation bias could (at least) partly account for the

368 inferior predictive performance of current computational models. 


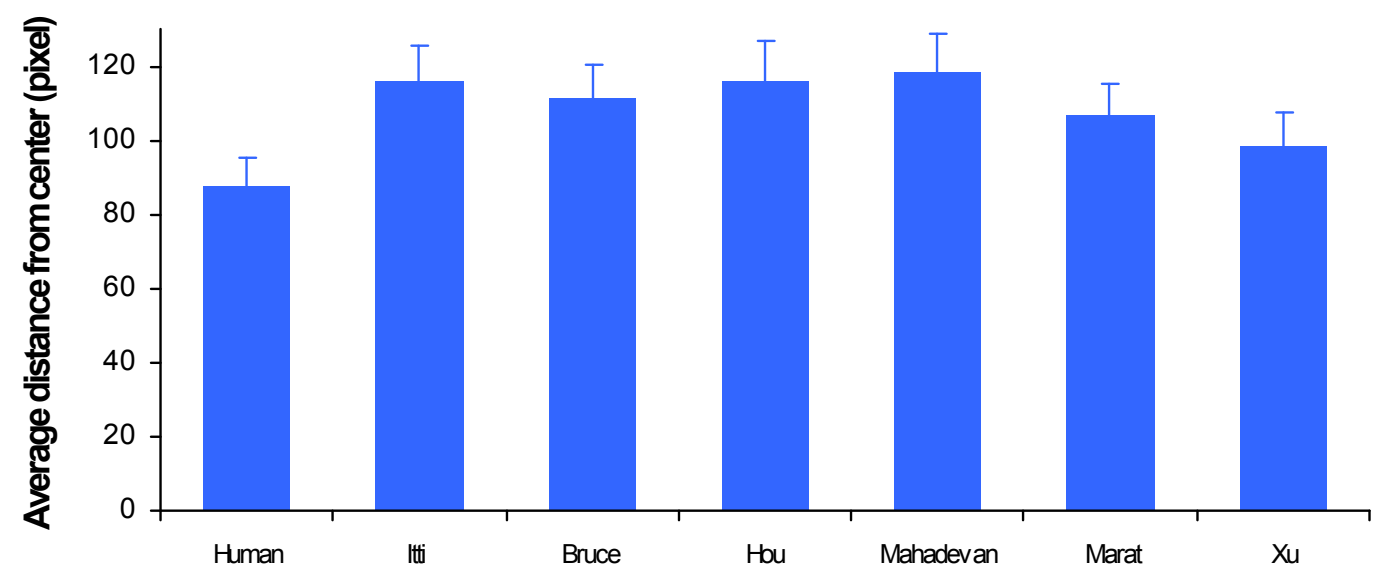

369

370 Figure 5. Comparison of actual and predicted fixation distribution in viewing of traffic videos, 371 using metrics of averaged fixation distance from the video frame centre. Error bars represent 372 standard error of the mean.

Discussion

In this eye-tracking study, we selectively disrupted the spatio-temporal regularity of

377 typical traffic videos, and compared actual human gaze allocation with predicted gaze allocation by state-of-the-art visual attention models. Our analysis clearly demonstrated a high human interobserver agreement for viewing the original and manipulated video clips (Fig. 2), suggesting a very similar attention allocation strategy across individuals in driving conditions. The tested computational visual attention models, however, performed poorly in comparison with human gaze behaviour. Specifically, the models showed indistinguishable gaze prediction irrespective of stimuli presentation sequence (Fig. 3), suggesting that the spatio-temporal regularity has not been appropriately implemented by these models. Furthermore, human gaze allocation demonstrated a clear central fixation bias that was much stronger than in the gaze distribution predicted by the models (Fig. 5). It seems that the poor performance of visual attention models in processing of 
387 dynamic scenes was at least due to the lack of consideration of spatio-temporal regularity and 388 central fixation bias.

389 As an inherent property of dynamic natural scenes, spatio-temporal regularity is routinely

390 exploited by the human visual system to effectively process visual inputs. For instance, in

391 driving situation we frequently expect that a particular object (e.g. a car in the motorway) will be

392 presented at a particular location and time because of the spatial and temporal structure of the

393 current scene, and prior knowledge of the visual world regularities (Guo et al., 2004).

394 Consequently, we can easily direct our attention to the expected object and/or location to

395 perform visual tracking or target analysis with increased perceptual sensitivity (Cunningham \&

396 Wallraven, 2009; Guo et al., 2004; Hall et al., 2010), and ignore those objects that have high

397 physical saliency but are not part of spatio-temporal regularity in dynamic scenes. For example,

398 while watching driving videos or actually driving a car, the experienced drivers allocate more

399 fixations to the front and centre view than to the dashboard, right side or left side view

400 (Nabatilan et al., 2012). They also tend to make predictive fixations by looking far ahead and

401 visually targeting objects or locations on the desired trajectory or future path (Kandil, Rotter, \&

402 Lappe, 2010; Lappi, Lehtonen, Pekkanen, \& Itkonen, 2013).

403 In this study, we further observed that in comparison with normal video sequence,

404 manipulating spatio-temporal regularity by selectively disrupting temporal (i.e. reversed video

405 sequence) or spatial frame order (i.e. normal image sequence) significantly altered human

406 observers' attention allocation (Fig. 3). This effect was more evident in the presentation of

407 reversed video sequences, suggesting a high sensitivity of the human visual attention system to

408 the temporal sequence of predictable dynamic scenes. Such sensitivity could optimize visual

409 processing efficiency and accuracy. Indeed, psychological studies have observed that the 
410 judgment of face gender, identity and expression in degraded or noisy presentation conditions is

411 significantly impaired when reversing the facial movement video or altering its natural motion

412 speed (Lander \& Bruce, 2000; Hill \& Johnston, 2001; Cunningham \& Wallraven, 2009).

413 Similarly, reversing the direction of movement impairs object recognition in dynamic settings

414 (Vuong \& Tarr, 2004; Wang \& Zhang, 2010). Clearly, spatio-temporal regularity plays a crucial

415 role in attention allocation and associated scene perception in natural vision.

416 When exploring static scene images, human natural gaze behaviour often shows a central

417 fixation bias, which can be ascribed to several factors. First, in photography, objects of interest

418 are most often placed in the centre of the image. Second, the image centre as point of fixation

419 could ensure rapid access to every point of interest in the image. It might therefore be

420 advantageous to start scene viewing in the centre. The motor bias, the tendency to make shorter

421 and more horizontal saccades, does not play a big role in central fixation bias (Tatler, 2007). This

422 central fixation bias is often incorporated in the visual attention models for processing static

423 scenes (Borji et al., 2014). When viewing dynamic driving videos, our observers also

424 demonstrated a similar 'central' fixation bias. They tended to allocate attention to the horizontal

425 plane close to eye level, and paid little attention to the model-predicted salient region above or

426 below the eye level. This finding is in agreement with the recent studies demonstrating a

427 tendency to maintain gaze near the centre of the display when watching a wide range of video

428 clips, such as natural or urban scenes, sports, cartoon and film clips (Le Meur et al., 2007; Berg

429 et al., 2009; Tseng et al., 2009; Dorr et al., 2010). It seems that central fixation bias is a

430 spontaneous human gaze behavior that is independent of image/video content and cognitive task.

431 Although using computationally different methods to compute local saliency, all the

432 tested visual attention models performed far below the human baseline, suggesting that they 
433 cannot realistically predict human attention allocation in viewing of (at least) dynamic driving

434 clips. Furthermore, for each video frame these models predicted the same local saliency region or

435 object irrespective of stimuli presentation sequence (i.e. normal video sequence vs reversed

436 video sequence vs normal image sequence), indicating that the video's spatio-temporal regularity

437 has not been appropriately implemented in the computational models. Considering that the

438 human visual system applies spatial-temporal regularity as part of top-down guidance for 439 determining gaze allocation, it seems that this factor, like other top-down factors such as

440 knowledge of scene context and image statistics distribution (Torralba et al., 2006; Judd et al.,

441 2009; Kanan et al., 2009; Goferman et al., 2012), should also be incorporated in a realistic

442 manner into the visual attention model to cope with predictable dynamic visual inputs, possibly

443 through visual motion sensitive neuron models (e.g. directional selective visual neuron models;

444 Rind, 1990) and/or depth sensitive neuron models (Rind \& Bramwell, 1996; Gabbiani \& Krapp,

445 2006; Yue \& Rind, 2006, 2013). It should be noted, however, that the driving video clips just 446 represent part of the visual motion signals in our natural environment. The nature of human

447 driving behaviour may create a strong central fixation bias and subsequently amplify its role in 448 visual saliency computation. The global optic flow associated with driving clips may also disrupt 449 those attention models designed to detect local featural motion. The extent to which we can 450 generalize current findings to other types of motion signals remains to be seen.

452 References

453 Acik A, Onat S, Schumann F, Einhauser W, Konig P (2009) Effects of luminance contrast and its 454 modifications on fixation behaviour during free viewing of images from different categories. $455 \quad$ Vision Res 49: 1541-1553.

456 Anderson BA (2013) A value-driven mechanism of attentional selection. Journal of Vision 457 13(3):7, 1-16. 
458 Ban S, Lee I, Lee M (2008) Dynamic visual selective attention model. Neurocomputing 71: 853459856.

460 Betz T, Kietzmann TC, Wilming N, Konig P (2010) Investigating task-dependent top-down 461 effects on overt visual attention. J Vis 19(3):15, 1-14.

462 Berg DJ, Boehnke SE, Marino RA, Munoz DP, Itti L (2009) Free viewing of dynamic stimuli by 463 humans and monkeys. J Vis 9(5):19, 1-15.

464 Borji A, Sihite DN, Itti L (2013) Quantitative analysis of human-model agreement in visual 465 saliency modelling: a comparative study. IEEE Trans Image Process 22: 55-69.

466 Borji A, Sihite DN, Itti L (2014) What/where to look next? modeling top-down visual attention 467 in complex interactive environments. IEEE Trans Syst Man Cybern 44: 523-538.

468 Bruce NDB, Tsotsos JK (2006) Saliency based on information maximization. Adv Neural Inf 469 Process Syst 18: 155-162.

470 Carmi R, Itti L (2006) Visual causes versus correlates of attentional selection in dynamic scenes. $471 \quad$ Vision Res 46: 4333-4345.

472 Cunningham DW, Wallraven C (2009) Dynamic information for the recognition of 473 conversational expressions. J Vis 9(13):7, 1-17.

474 Dorr M, Martinetz T, Gegenfurtner KR, Barth E (2010) Variability of eye movements when 475 viewing dynamic natural scenes. J Vis 10(10):28, 1-17.

476 Einhäuser W, Rutishauser U, Frady EP, Nadler S, König P, Koch C (2006) The relation of phase 477 noise and luminance contrast to overt attention in complex visual stimuli. J Vis 6: 1148$478 \quad 1158$.

479 Gabbiani F, Krapp HG (2006) Spike-frequency adaptation and intrinsic properties of an 480 identified looming sensitive neuron. J Neurophysiol 96: 2951-2962.

481 Gavin CJ, Houghton S, Guo K (2015) Dog owners show experience-based viewing behaviour in 482 judging dog face approachability. Psychol Res doi:10.1007/s00426-015-0718-1

483 Goferman S, Zelnik-Manor L, Tal A (2012) Context-aware saliency detection. IEEE Trans 484 Pattern Anal Mach Intell 34:1915-1926.

485 Green D, Swets J (1966) Signal detection theory and psychophysics. New York: John Wiley.

486 Guo K, Mahmoodi S, Robertson RG, Young MP (2006) Longer fixation duration while viewing 487 face images. Exp Brain Res 171: 91-98. 
488 Guo K, Nevado A, Robertson RG, Pulgarin M, Thiele A, Young MP (2004) Effects on 489 orientation perception of manipulating the spatiotemporal prior probability of stimuli. Vision $490 \quad$ Res 44: 2349-2358.

491 Guo K, Smith C, Powell K, Nicholls K (2012) Consistent left gaze bias in processing different 492 facial cues. Psychol Res 76: 263-269.

493 Hall S, Pollux PMJ, Guo K (2010) Exploitation of natural geometrical regularities facilitates 494 target detection. Vision Res 50: 2411-2420.

495 Henderson JM (2007) Regarding scenes. Curr Dir Psychol Sci 16: 219-222.

496 Hou X, Zhang L (2008) Dynamic visual attention: Searching for coding length increments.

497 In Advances in neural information processing systems (pp. 681-688). MIT Press.

498 Hill H, Johnston A (2001) Categorizing sex and identity from the biological motion of faces. 499 Curr Biol 11: 880-885.

500 Itti L, Koch C (2000) A saliency-based search mechanism for overt and covert shifts of visual 501 attention. Vision Res 40: 1489-1506.

502 Itti L, Koch C, Niebur E (1998) A model of saliency-based visual attention for rapid scene 503 analysis. IEEE Trans Pattern Anal Mach Intell 20: 1254-1259.

504 Judd T, Ehinger K, Durand F, Torralba A (2009) Learning to predict where humans look. IEEE 505 12th International Conference on Computer Vision 2106-2113.

Kanan C, Tong MH, Zhang L, Cottrell GW (2009) SUN: Top-down saliency using natural 507 statistics. Vis Cogn 17: 979-1003.

508 Kandil F, Rotter A, Lappe M (2010) Car drivers attend to different gaze targets when negotiating 509 closed vs. open bends. J Vis 10(4):24, 1-11.

510 Lander K, Bruce V (2000) Recognizing famous faces: Exploring the benefits of facial motion. 511 Ecol Psychol 12: 259-272.

512 Lappi O, Lehtonen E, Pekkanen J, Itkonen T (2013) Beyond the tangent point: Gaze targets in 513 naturalistic driving. J Vis 13(13):11, 1-18.

514 Le Meur O, Le Callet P, Barba D (2007) Predicting visual fixations on video based on low level 515 visual features. Vision Res 47: 2483-2498.

516 Ma Y, Hua X, Lu L, Zhang H (2005) A generic framework of user attention model and its 517 application in video summarization. IEEE Trans Multimedia 17: 907-920. 
518 Mahadevan V, Vasconcelos N (2010) Spatiotemporal saliency in dynamic scenes. IEEE Trans

519 Pattern Anal Mach Intell 32: 171-177.

520 Mannan SK, Ruddock KH, Wooding DS (1996) The relationship between the locations of spatial

521 features and those of fixations made during visual examination of briefly presented images.

522 Spat Vis 10: 165-188.

523 Marat S, Phuoc TH, Granjon L, Guyader N, Pellerin D, Guèrin-Duguè A (2009) Modelling

524 spatio-temporal saliency to predict gaze direction for short videos. Int J Comput Vis 82: 231525243.

526 Nabatilan LB, Aghazadeh F, Nimbarte AD, Harvey CC, Chowdhury SK (2012) Effect of driving 527 experience on visual behavior and driving performance under different driving 528 conditions. Cogn Technol Work 14: 355-363.

529 Parkhurst DJ, Niebur E (2003) Scene content selected by active vision. Spat Vis 16: 125-154.

530 Pollux PMJ, Guo K (2009) Event-related potential correlates of spatiotemporal regularities in $531 \quad$ vision. NeuroReport 20: 525-530.

532 Pollux PMJ, Hall S, Guo K (2014) Facial expression training optimises viewing strategy in 533 children and adults. PLoS One 9(8), e105418.

534 Pollux PMJ, Hall S, Roebuck H, Guo K (2011) ERP correlates of the interaction between 535 attention and spatiotemporal context regularity in vision. Neurosci 190: 258-269.

536 Reinagel P, Zador AM (1999) Natural scene statistics at the centre of gaze. Network-Comp 537 Neural 10: 341-350.

538 Rind FC (1990) A directionally selective motion-detecting neurone in the brain of the locust: 539 physiological and morphological characterization. J Exp Biol 149: 1-19.

540 Rind FC, Bramwell DI (1996) Neural network based on the input organization of an identified neuron signalling impending collision. J Neurophysiol 75: 967-985.

542 Roebuck H, Bourke P, Guo K (2014) Role of lateral and feedback connections in primary visual 543 cortex in the processing of spatiotemporal regularity? a TMS study. Neurosci 263: 231-239.

544 Röhrbein F, Goddard P, Schneider M, James G, Guo K (2015) How does image noise affect 545 actual and predicted human gaze allocation in assessing image quality? Vision Res 112: 1154625.

547 Rothenstein A, Tsotsos J (2006) Attention links sensing to recognition. Image Vision Comput 26: $548 \quad 114-126$. 
549 Tatler BW (2007) The central fixation bias in scene viewing: Selecting an optimal viewing 550 position independently of motor biases and image feature distributions. J Vis 7(14):4, 1-17.

551 Tatler BW, Hayhoe MM, Land MF, Ballard DH (2011) Eye guidance in natural vision:

552 Reinterpreting salience. J Vis 11(5):5, 1-23.

553 Torralba A, Oliva A, Castelhano MS, Henderson JM (2006) Contextual guidance of eye 554 movements and attention in real-world scenes: The role of global features in object search. 555 Psychol Rev 113: 766-786.

556 Tseng PH, Carmi R, Cameron IG, Munoz DP, Itti L (2009) Quantifying center bias of observers 557 in free viewing of dynamic natural scenes. J Vis 9(7):4.

558 Vuong QC, Tarr MJ (2004) Rotation direction affects object recognition. Vision Res 44: 1717$559 \quad 1730$.

560 Wang Y, Zhang K (2010) Decomposing the spatiotemporal signature in dynamic 3D object 561 recognition. J Vis 10(10):23, 1-16.

$562 \mathrm{Xu}$ J, Yue S, Tang Y (2013) A motion attention model based on rarity weighting and motion 563 cues in dynamic scenes. Int J Pattern Recog 27(6): 1355009.

$564 \mathrm{Xu} \mathrm{J}$, Yue S (2014) Mimicking visual searching with integrated top down cues and low-level 565 features. Neurocomputing 133: 1-17.

566 Yue S, Rind FC (2006) Collision detection in complex dynamic scenes using a LGMD based 567 visual neural network with feature enhancement. IEEE Trans. Neural Networks 17: 705-716.

568 Yue S, Rind FC (2013) Redundant neural vision systems - competing for collision recognition 569 roles. IEEE Trans Auton Ment Dev 5: 173-186.

570 Zou KH, O'Malley AJ, Mauri L (2007) Receiver-operating characteristic analysis for evaluating 571 diagnostic tests and predictive models. Circulation 115: 654-657.

572

573

574 575 